\title{
Similarity attraction-based mobile advertising: designing and testing
}

\author{
$M \operatorname{Shin}^{1}$, and $K W \mathrm{Lee}^{2, *}$ \\ ${ }^{1}$ Department of Herb Science, Dong-Eui Institute of Technology, Busan 47230, Korea \\ ${ }^{2}$ Hallym University Sacred Heart Hospital, Gwanpyeong-ro, Dongan-gu, An-yang, South Korea
}

\begin{abstract}
This study explores the possibility of applying the sociopsychological phenomenon known as similarity attraction to the creation of more customized and persuasive mobile advertising without privacy concerns, and for this purpose, we develop two applications, SensPlus and PerAds. While the SensPlus app accumulates the smartphone usage profiles of participants and constructs a regression model to predict their personality, the PerAds app presents user-created content according to the user's personality. The participants' response to a smartphone ad is measured in terms of invitation acceptance of the cherry blossom festival and classical music concert. The $\chi^{2}$ statistics imply participants respond differentially to the various types of smartphone ads according to the similarity between the personalities of the user and ad types. Our results indicate that the socio-psychological phenomenon can be introduced into practical application by replacing the personality trait factors with the smartphone usage pattern.
\end{abstract}

\section{Introduction}

The advertisement landscape is undergoing rapid changes due to the decline in nonindividualistic mass communication and rise in customized and personalized communication. Smartphones are at the core of this change. However, most of current customized advertising strategies like ads based on user's location [1], internet search [2] and personal profiles [3], [4] paid little attention to socio-psychological aspects.

We investigate the possibility of a customized advertising strategy based on the interaction between smartphone usage profiles and ad types, known as the "similarityattraction effect," that is, the tendency of people to be attracted by those similar to themselves [5], [6]. Most of the similarity-attraction studies based on personality employ personality questionnaire, but in real-world we cannot ask users to complete a personality questionnaire [7]. Smartphones can provide us with sufficient information to extract or infer the users' psychological states or personality traits [8], [9].

In this study, we test the opportunity by designing a smartphone ads system in which (1) the smartphone user's personality is based on smartphone usage pattern and not a questionnaire response; (2) the presentation of smartphone ads is customized to suit the

\footnotetext{
* Corresponding author: kangwooster@gmail.com
} 
smartphone user's personality; and (3) the smartphone user's personality information should not be collected, implying that the privacy-concern should be reduced.

\section{System model and methods}

\subsection{Personality-based smartphone ads system}

Some text. The general scheme of the personality-based smartphone ads system is presented in figure 1. The scheme consists of two parts, regression modeling and customized advertisement.

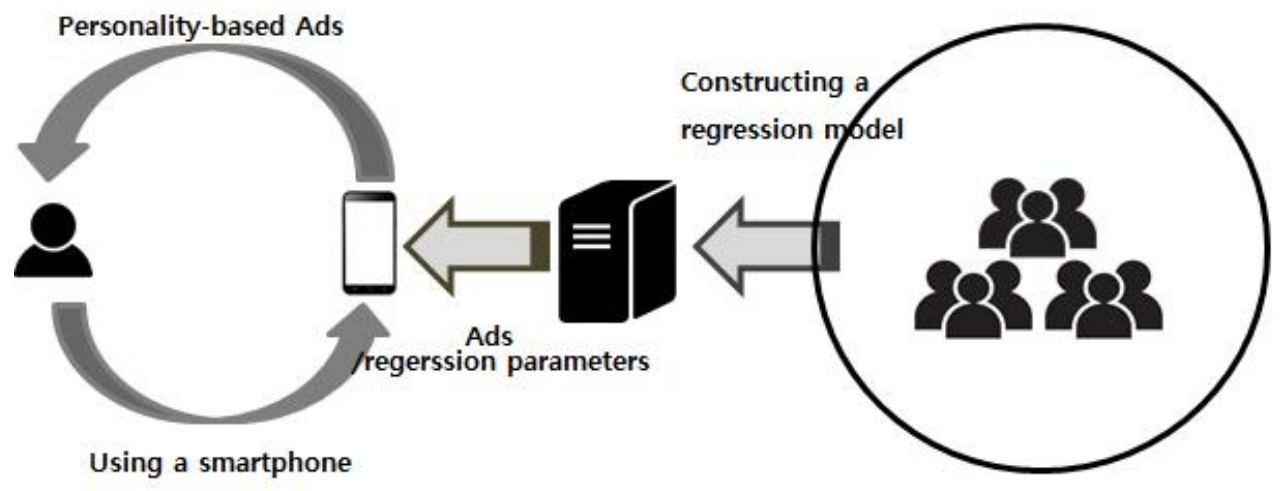

Fig. 1. General scheme of the personality-based smartphone ads system.

Our previous study [10] constructed a regression model using the SensPlus app and support vector machine (SVM) regression. The SensPlus app collects smartphone usage profiles such as number and time of incoming and outgoing calls and number of SMSs (input), and then transfers them to a server. A regression model can be established by mapping the smartphone usage profile to the extroversion trait (output), a modified version of the Wiggins personality test.

For customized advertisement, the PerAds app which selectively presents personalitybased ads was developed on the Android platform. Two different types of ads are used one to promote extroversion, and the other to promote introversion. The decision of which type of ad is presented to the user depends on the threshold values set by the experimenter. To avoid misclassification, we used dual thresholds $T_{1}$ and $T_{2}$. The ad is short and can be interrupted by the user anytime. The detailed procedure of PerAds is given below:

- Accumulate the user's smartphone usage profiles and average the profiles.

- Calculate the user's extroversion value by setting the averaged smartphone usage profile in the regression model.

- Compare the estimated extroversion value with the threshold value.
value $>T_{1} \quad:$ extrovert
value $<T_{2} \quad$ : introvert
$T_{2} \leq$ value $\leq T_{1}:$ ambivert

\subsection{Methods}

\subsubsection{Participants}

A total of 176 undergraduate and graduate students with an Android smartphone enrolled in the experiment and paid 10,000 Korean won (approximately USD 9). They were aged from 
20 to 35 years, with an average age of 28.7 ( $\mathrm{SD}=6.7$ years). They signed a consent form and were informed of the experiment procedure.

\subsubsection{PerAds apps}

Two animation video clips (figure 2) were used for the experiment, one to advertise a cherry blossom festival for extroverted participants and the other to advertise a classical music concert for introvert participants. An animated avatar introduces each event and invites a smartphone user. In order to create the extrovert and introvert ads, three visual cues of the avatars were manipulated, that is, clothe types, movement, and invitation phrases. The extrovert avatar wore a casual suit, actively moved her body, and used informal language, whereas the introvert avatar wore a formal suit, rarely moved her body, and used formal language. Each video clip had a 320 × 240 pixel resolution and lasted five seconds. Text-only ads were also used that contained information such as "invitation for cherry blossom festival (for extroverts)," "invitation for classical music concert (for introverts)," date, and location. The date and location are the same for both the extrovert and introvert clips.
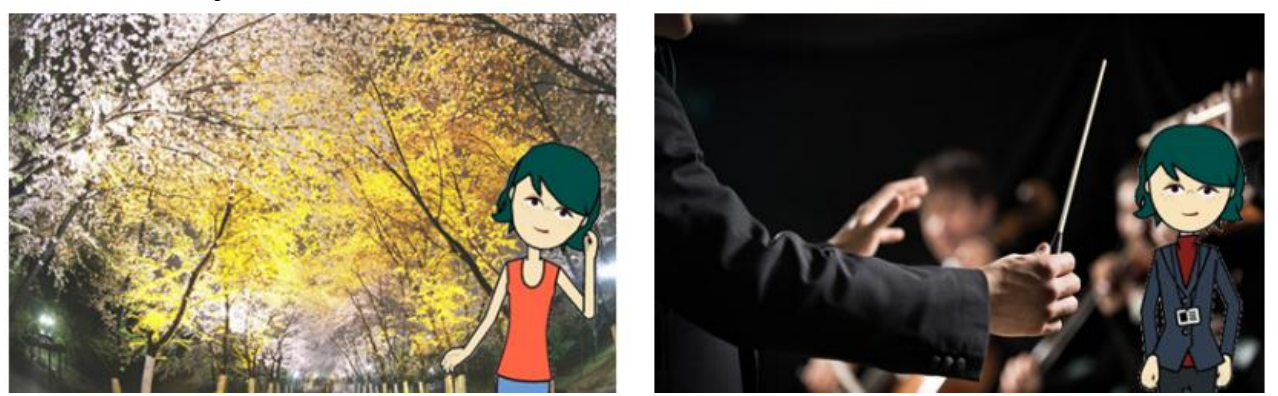

Fig. 2. The animation video clips used for advertising stimulus. (a) Invitation for cherry blossom festival (b) Invitation for classical music concert.

\subsubsection{Procedure}

The participants were randomly assigned to two groups, the text-only group (80 participants) and the animation group (96 participants). Text-only ads were given to the text-only group, and animation ads were given to the animation group. The participants were asked to install the mobile app PerAds in their phone, with instruction not to delete the application until 15 days later, that is, until the end of the experiment period. One day after the usage data accumulation period, an invitation ad is presented differentially to each participant according to his/her estimated personality. After the participants watched the ads, they are asked whether they would accept the invitation or not. The responses are sorted by the invitation and media type.

\section{Results}

We carried out chi-square analysis for each group using the number of participants who responded "accept" or "reject" to the invitation of events. The participants who did not indicate either extroverted or introverted traits (between $T_{1}$ and $T_{2}$ ) were not included in the statistical analysis. The results are summarized in tables 1,2 , and 3.

For the text-only group, we expected number of accept (or reject) as $50 \%$ of participants, because we did not expect any difference between accept and reject responses to the invitations. The result is given in table 1 . For the festival condition, the chi-square $\left(\chi^{2}\right)$ 
statistic is 1.324 and the p-value is 0.2498 . For the concert condition, the chi-square is 0.533 and the $\mathrm{p}$-value is 0.4652 . Both results are not statically significant at $p<.05$.

Table 1. Chi-square results for the participants' responses to the cherry festival invitation (top) and the classical music concert invitation (bottom) of text-only stimulus (1).

\begin{tabular}{|c|c|c|c|}
\hline Festival & Observed & Expected \# & Expected \\
\hline Accept & 22 & 18.5 & $50 \%$ \\
Reject & 15 & 18.5 & $50 \%$ \\
\hline Column Totals & 37 & 37 & $100 \%$ \\
\hline \hline Concert & Observed & Expected \# & Expected \\
\hline Accept & 17 & 15 & $50 \%$ \\
Reject & 13 & 15 & $50 \%$ \\
\hline Column Totals & 30 & 30 & $100 \%$ \\
\hline
\end{tabular}

For the animation group, we again set the expected number of accept (or reject) to half the number of each personality trait, and then carried out the chi-square analysis. The result is given in Table 2. For the festival condition, the chi-square statistic is 6.422 and the pvalue is 0.0113 . This result is statistically significant at $p<.05$. However, for the concert condition, the chi-square is 3.457 and the $p$-value is 0.0630 .

Table 2. Chi-square results for the participants' responses to the cherry festival invitation (top) and the classical music concert invitation (bottom) of animated stimulus (2).

\begin{tabular}{|c|c|c|c|}
\hline Festival & Observed & Expected \# & Expected \\
\hline Accept & 31 & 22.5 & $50 \%$ \\
Reject & 14 & 22.5 & $50 \%$ \\
\hline Column Totals & 45 & 45 & $100 \%$ \\
\hline \hline Concert & Observed & Expected \# & Expected \\
\hline Accept & 23 & 17.5 & $50 \%$ \\
Reject & 12 & 17.5 & $50 \%$ \\
\hline Column Totals & 35 & 35 & $100 \%$ \\
\hline
\end{tabular}

The assumption of no difference between 'accept' and 'reject' responses to the invitations can be problematic. For the text-only condition, though the ad stimulus is relatively neutral, the participants are more likely to accept the invitation, but the tendency is not statistically significant. This reflects the participants' attitude or response tendency to the events and can be considered as the baseline for the effect of animated advertising. Thus, we re-calculate the expected numbers by multiplying the total number of participants in each condition with the proportion of each response in the text-only group: $45 \times 22 / 37(26.775), 45 \times 15 / 37(18.225)$ for the festival invitation, $35 \times 17 / 30(19.845)$, $35 \times 13 / 30(15.155)$ for the concert invitation. The result is given in Table 3. For the festival condition, the chi-square statistic is 1.646 and the p-value is 0.1995 . For the concert condition, the chi-square is 1.158 and the p-value is 0.2818 . Both results are not statistically significant at $p<.05$. 
Table 3. Chi-square results for the participants' responses to the cherry festival invitation (top) and the classical music concert invitation (bottom) of text-only stimulus (3).

\begin{tabular}{|c|c|c|c|}
\hline Festival & Observed & Expected \# & Expected \\
\hline Accept & 31 & 26.775 & $59.5 \%$ \\
Reject & 14 & 18.225 & $40.5 \%$ \\
\hline Column Totals & 45 & 45 & $100 \%$ \\
\hline \hline Concert & Observed & Expected \# & Expected \\
\hline Accept & 23 & 19.845 & $56.7 \%$ \\
Reject & 12 & 15.155 & $43.3 \%$ \\
\hline Column Totals & 35 & 35 & $100 \%$ \\
\hline
\end{tabular}

\section{Discussion and conclusion}

The scheme of personality-based ads that use smartphone usage profiles to estimate the user's personality not requiring personal information by separating regression modeling from personality estimation was proposed. Furthermore, we suggest the non-privacy invasive evaluation procedure to test the effect of the scheme. Usual $2 \times 2$ factorial design allows for investigating the interaction between independent factors, but needs to know the properties of individuals that are privacy-sensitive. Thus, the factorial design is impractical for a real world application. In contrast, our experiment design does not require privacysensitive information of participants and can thus be applied more freely to smartphone application.

Throughout the experiment, we tried to demonstrate the effect of the personality-based ad scheme. Our results indicate that the participants are more likely to accept than reject the invitation. This study introduces the socio-psychological phenomenon of similarity attraction to the customized advertisement application domain by establishing the relationship between extroversion and smartphone usage pattern. However, we could not get significant difference of acceptance rate using animated ads from acceptance rate using text-only ads. That might be due to the quality of ads. Advertisement is field of art, so acceptance rate might be more influenced by sense, concept, graphic etc. Further try with more expressive animated ads is expected.

\section{Acknowledgments}

This research was supported by Basic Science Research Program through the National Research Foundation of Korea (NRF) funded by the Ministry of Education (2016R1A6A3A11933734).

\section{References}

1. U. Varshney, "Location management for mobile commerce applications in wireless internet environment", ACM Transactions on Internet Technology, vol. 3, no. 3, pp.236-255, 2003.

2. K. Partridge, B. Begole, P. Alto and C. H. Road, "Activity-based Advertising: Techniques and Challenges", in Proc of the First Workshop on Pervasive Advertising, May 11, pp. 2-5, 2009. 
3. D. J. Xu, S. S. Liao and Q. Li, "Combining empirical experimentation and modeling techniques: A design research approach for personalized mobile advertising applications", Decision Support Systems, vol. 44, no. 3, pp. 710-724, 2008.

4. J. B. Todd, Y. Christine and S. Esther, "From firm-controlled to consumer-contributed: consumer co-production of personal media marketing communication", Journal of Interactive Marketing, vol. 28, no. 2, pp.117-133, 2014.

5. E. C. Klohnen and S. Luo, "Interpersonal attraction and personality: What is attractive - self similarity, ideal similarity, complementarity, or attachment security?", Journal of Personality and Social Psychology, vol. 85, no. 4, pp. 709-722, 2003.

6. M. Matthew, S. H. Robert and K. Jeffrey, "Is actual similarity necessary for attraction? A meta-analysis of actual and perceived similarity", Journal of Social and Personal Relationships, vol. 25, no. 6, pp. 889-922, 2008

7. F. Evanthia, T. Athanasios and T. Giannis, "An integrated e-recruitment system for automated personality mining and applicant ranking", Internet Research, vol. 22, no. 5, pp.551-568, 2012.

8. W. Lane and C. Manner, "The Impact of Personality Traits on Smartphone Ownership and Use", International Journal of Business and Social Science, vol. 2, no. 17, pp. 2228, 2003.

9. S. Butt and J. G. Phillips "Personality and self reported mobile phone use," Computers in Human Behavior, vol. 7, no. 3, pp. 346-360, 2008.

10. K. Lee. and H. Choo, "Predicting user attitudes toward smartphone ads using support vector machine", International Journal of Mobile Communications, vol. 14, no. 3, pp. 226-243, 2016. 